\title{
Optically-Generated Many Spin Entanglement in a Quantum Well
}

\author{
J. Bao* ${ }^{*}$, A. V. Bragas ${ }^{* \dagger}$, J. K. Furdyna** and R. Merlin* \\ *FOCUS Center and Department of Physics, University of Michigan, Ann Arbor, MI 48109-1120, USA \\ "Present address: Division of Engineering and Applied Sciences, Harvard University, Cambridge, MA 02138, USA \\ ${ }^{\dagger}$ Present address: FCEyN, Universidad de Buenos Aires, Ciudad Universitaria, 1428 Buenos Aires, Argentina. \\ ${ }^{* *}$ Department of Physics, University of Notre Dame, Notre Dame, IN 46556, USA
}

\begin{abstract}
A many-particle system may exhibit non-local behavior in that measurements performed on one of the particles can affect a second particle that is far apart. These so-called entangled states are essential for the implementation of quantum information protocols and gates for quantum computation. Here, we use ultrafast optical pulses and coherent techniques to create spin entangled states of non-interacting electrons bound to donors in a CdTe quantum well.
\end{abstract}

\section{INTRODUCTION}

The problem of quantum entanglement has attracted much attention since the early days of quantum mechanics. Following the proposal by Deutsch for a quantum computer in 1985, the building of a quantum cryptography machine by Bennett et al. in 1989, and the discoveries by Shor in 1994 and by Grover in 1996 of quantum algorithms, the question of entanglement has now acquired practical significance [1]. Here, we propose and demonstrate experimentally a novel method for many-particle entanglement involving spin states of non-interacting paramagnetic impurities in a quantum-well (QW) [2].

\section{QUBITS AND ENTANGLEMENT}

Our qubits are embodied by Zeeman-split spin states of donor-bound electrons in a $\mathrm{Cd}_{1-x} \mathrm{Mn}_{x} \mathrm{Te} \mathrm{QW}$ [2]. The $N$-electron entangled states relevant to our work are coherent superpositions of the form

$$
\psi=\sum_{k=0}^{2 S} C_{k} e^{-i k \Omega_{e^{t}}}|S-k\rangle
$$

where $|S-k\rangle$ is an eigenstate of the Zeeman energy operator with $S=N / 2, \Omega_{0}=g \mu_{\mathrm{B}} B / \quad$ ( $g$ is the gyromag- netic factor and $B$ is the external magnetic field), and $C_{k}$ are parameters that can be controlled by optical means [2]. Our entanglement method relies on the exchange coupling between the hole of the photoexcited exciton and the donor electrons. This interaction can be described in terms of an effective magnetic field $\mathbf{B}_{\mathrm{e}}=\kappa \mathbf{J}$ where $\mathbf{J}$ is the spin of the heavy-hole and $\kappa$ is an exchange constant. Due to a combination of spinorbit-coupling and quantum-confinement effects, the heavy-hole spin and, consequently, $\mathbf{B}_{\mathrm{e}}$ are parallel to the QW growth axis (the $z$-axis) at small external fields [3]. Thus, $J_{z}= \pm 3 / 2$ and, provided the external field is not parallel to the same axis, the quantization axes in the absence and in the presence of the exciton are different and, since the exciton does not modify $S$, the corresponding states are not orthogonal to each other. It follows that, for an electric-dipole allowed exciton, Raman coherences between arbitrary states can be established by using two laser fields tuned to resonate with the exciton energy, $E_{\mathrm{e}}$, so that their frequency difference is $|m-l| \Omega_{0}$.

As it is well known for generic Raman coherences [4], we expect in the limit $\Omega_{0}<<E_{\mathrm{e}}$ that the effect of the coherent spin states of Eq. (1) on the optical properties be that of a modulation involving the harmonics of $\Omega_{0}$. This modulation can be probed in the time domain using standard ultrafast pump-probe methods [2]. We notice that overtones of $\Omega_{0}$ should not be observed 
for non-interacting electrons, and that, because the electron spin is $s=1 / 2$, the signature for an entanglement of $N$ electrons is the observation of the $N$ th harmonic.

\section{EXPERIMENT}

Our sample consists of 100 periods of 58 - $\AA$-thick $\mathrm{Cd}_{1-x} \mathrm{Mn}_{x} \mathrm{Te}(x=0.0039)$ with $19-\AA \AA$-thick MnTe barriers grown by molecular beam epitaxy on a CdTe substrate along the [001] $z$-axis. The sample is nominally undoped. However, spin flip Raman experiments reveal the presence of isolated donors in the wells (possibly indium) with a concentration of $\sim 5 \times 10^{16} \mathrm{~cm}^{-3}$.

In our time-domain experiments, we used circularly-polarized pump pulses to generate the Raman coherences which we probed by measuring the pumpinduced shift of the polarization angle of the reflected probe field, $\Delta \theta$ (magnetic-Kerr effect) [2]. Our modelocked Ti-sapphire laser provided $\sim 130 \mathrm{fs}$ pulses of central energy $\omega_{\mathrm{C}}$ in the range $1.47-1.72 \mathrm{eV}$ $(720-840 \mathrm{~nm})$ at a repetition rate of $82 \mathrm{MHz}$.

Time-domain data for $\omega_{\mathrm{C}}$ slightly above the QW gap is shown in Fig. 1. At short times, the signal is dominated by electron Zeeman quantum beats, i. e., oscillations showing a field- and temperature-behavior consistent with that of spin-flips of electrons [2]. The oscillations that persist above $\sim 15 \mathrm{ps}$ are associated with the paramagnetic resonance (PR) of the $\mathrm{Mn}^{2+}$ ions with $g \approx 2$. The inset shows the Fourier decomposition spectrum with parameters gained from linear prediction fits (the PR is not shown) [2]. In addition to the peak labeled 1SF, associated with single-electron spinflips, the data reveal features $2 \mathrm{SF}$ and $3 \mathrm{SF}$, at nearly twice and three times the frequency of 1SF, which are assigned to multiple spin-flips of donors. The relatively large spectral width of these lines is ascribed to inhomogeneous broadening arising from fluctuations in the manganese concentration [2]. The observation of two spin-flip overtones indicates the establishment of Raman coherences and, hence, entanglement involving at least three donor impurities. As discussed in [2], the spin-flip harmonics as well as the $\mathrm{Mn}^{2+}$-PR exhibit a significant enhancement when $\omega_{\mathrm{C}}$ is tuned to resonate with localized exciton states below the gap. Excitation at energies close to but above the QW gap, as in Fig. 1, leads to an enhancement of the signal of the overtones with respect to the fundamental. These results clearly indicate that the bound-electron entanglement benefits from the mediation of states in the continuum. However, the process by which the entan-

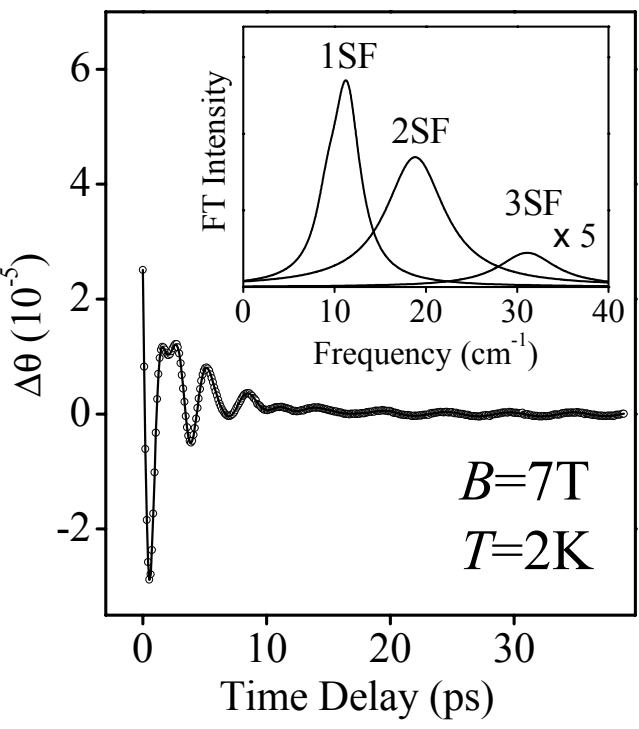

FIGURE 1. Differential magnetic Kerr data at $\omega_{\mathrm{C}}=$ $1.71 \mathrm{eV}$. Inset: Fourier decomposition spectrum.

glement is attained is not well understood. We note that a mechanism involving the RKKY interaction between localized electrons and delocalized excitons has been previously proposed [5].

\section{ACKNOWLEDGMENTS}

Work supported by the NSF under Grants No. PHY 0114336 and No. DMR 0245227, and by the DARPASpinS program. Acknowledgment is made to the donors of The Petroleum Research Fund, administered by the ACS, for partial support of this research.

\section{REFERENCES}

1. See: M. A. Nielsen and I. L. Chuang, Quantum Computation and Quantum Information (University Press, Cambridge, 2000); and references therein.

2. J. M. Bao, A. V. Bragas, J. K. Furdyna, and R. Merlin, Nature Mater. 2, 175 (2003); Solid State Commun. 127, 771 (2003).

3. R. W. Martin, R. J. Nicholas, G. J. Rees, S. K. Haywood, N. J. Mason, and P. J. Walker, Phys. Rev. B 42, 9237 (1990).

4. R. W. Boyd, Nonlinear Optics. (Academic Press, San Diego, 1992) Ch. 9, pp. 365-397.

5. C. Piermarocchi, P. Chen, L. J. Sham and D. G. Steel, Phys. Rev. Lett. 89, 167402 (2002). 